\title{
Reinforced Deep Learning for Verifying Finger Veins
}

\author{
$\underline{\text { https://doi.org/10.3991/ijoe.v17i07.24655 }}$ \\ Shaima Miqdad Mohamed Najeeb, Raid Rafi Omar Al-Nima, \\ Mohand Lokman Al-Dabag ${ }^{(\varpi)}$ \\ Northern Technical University, Technical Engineering College, Mosul, Iraq \\ mohandaldabagentu.edu.iq
}

\begin{abstract}
Recently, personal verifications become crucial demands for providing securities in personal accounts and financial activities. This paper suggests a new Deep Learning (DL) model called the Re-enforced Deep Learning (RDL). This approach provides another way of personal verification by using the Finger Veins (FVs). The RDL consists of multiple layers with a feedback. Two FV fingers are employed for each person, $\mathrm{FV}$ of the index finger for first personal verification and $\mathrm{FV}$ of the middle finger for re-enforced verification. The used database is from the Hong Kong Polytechnic University Finger Image (PolyUFI) database (Version 1.0). The result shows that the proposed RDL achieved a promising performance of $91.19 \%$. Also, other DL approaches are exploited for comparisons in this study including state-of-the-art models.
\end{abstract}

Keywords_-Finger Veins, Verification, Deep Learning

\section{Introduction}

Biometrics have become important for the roles of personal information securities. There are many biometric characteristics such as fingerprint, face, voice, palm, iris, Finger Texture (FT) and Finger Vein (FV). The FV recognition has received significant attentions and it is highly safe to be utilized. It is a technique of pattern recognition based on personal FV pattern under a finger skin's surface.

Each finger of each individual has a unique FV pattern so, it differs even between identical twins and even between personal fingers. Furthermore, these patterns remain stable along the human's life [1]. FVs are located inside skin, therefore, is a tiny risk of getting hacked or forgery. Also, rough and rash skins do not affect the recognition rates. Finally, FVs are less affected by the variations of physical conditions such as weather [2], however, they are hard to capture comparing to other biometric characteristics [3] [4] [5].

In the literature, several studies have adopted FV and deep learning (DL) for person authentication. Liu et al. used a Convolution Neural Network (CNN) for FV recognition. The $\mathrm{CNN}$ has seven layers, five of them are convolutional layers and the rest are fully-connected layers. The authors acquired a new dataset and used benchmarked datasets to check the suggested method. A comparison was also made with the traditional method to figure out how the proposed method attained the best results [6]. Boucherit 
et al. claimed that they had good results after using an improved DL network known as the Merge Convolutional Neural Network (Merge CNN). Three datasets were also used in this work to support the proposed method's results [7]. Zeng et al. proposed FV verification algorithm depending on Fully Convolutional Neural Network (FCN) and Conditional Random Field (CRF). The performance of the proposed method was tested by utilizing more than one benchmarked dataset. Traditional back-propagation algorithm was integrated in the training process of the FCN [8]. Shendre and Sapkal used a hybrid DL method to recognize the FV based on the Binary Decision Diagram (BDD) and fuzzy commitment schemes. Four stages were applied: FV feature extraction, template generation (BDD-based), fuzzy involvement scheme, recognition and decision making based on the machine learning. A comparison was made in this paper and the suggested method gave acceptable results compared to other methods [9]. Yang et al. proposed a method called the Binary Decision Diagram-Multi-Layer Extreme Learning Machine (BDD-ML-ELM) to create a non-invertible FV prediction system. Two datasets were utilized for evaluating the performance of the suggested method. A comparison was made to support the work which shows acceptable results compared to other methods [10].

This work aims to approach a new DL model termed the RDL. This method effectively utilizes multiple data of FVs to enhance the performance of personal verification.

The remaining sections of this paper are organized as follows: section 2 describes the proposed RDL, section 3 provides results and discussions, and section 4 declares the conclusion.

\section{Re-enforced Deep Learning (RDL)}

The suggested RDL is fundamentally a CNN with a global feedback. It is designed for personal verification, where an individual claims his/her identity and this claiming is approved or rejected. Figure 1 illustrates the general architecture of the proposed RDL approach. It consists of six layers, global feedbacks and two sets of weights. The layers are: convolution, Rectified Linear Unit (ReLU), pooling, Fully Connected (FC), softmax and classification layers. The global feedback is broadcasted from the last layer (the classification layer) to all previous hidden layers. The two sets of weights are for the FVs of index fingers and for the FVs of middle fingers. This network is motivated from the novel Re-enforced Probabilistic Neural Network (RPNN) which is approached in [11]. However, the RPNN is an Artificial Neural Network (ANN) and the proposed RDL is a new DL network version. To the best of the obtained knowledge, this is the first time of suggesting such model of the RDL. The following subsections provides brief descriptions for the RDL layers and its feedback. 


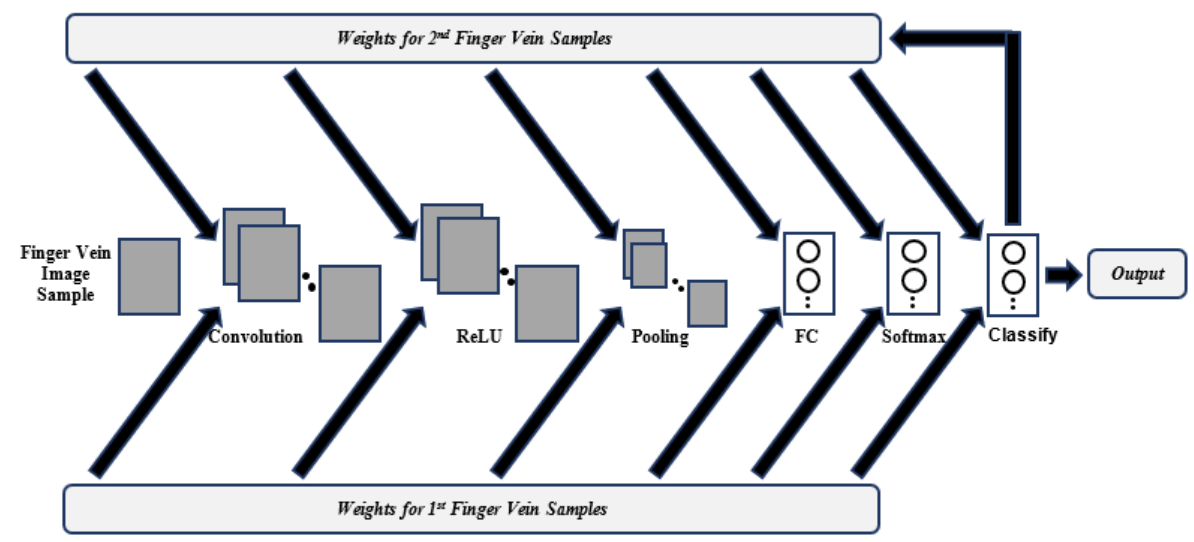

Fig. 1. Block diagram of RDL

\subsection{Convolutional layer (CL)}

This layer convolves the input image (FV) with its kernels of weights to extract image feature maps. In general, this layer has multiple channels with specific kernel (filter) size. The following equation represents the convolving equation in this layer:

$$
P_{n, m, c}^{l a}=B_{c} l a+\sum_{i=-k_{l}^{l a}}^{k_{l}^{l a}} \sum_{j=-k_{w}^{l a}}^{k_{w}^{l a}} \sum_{c^{l a-1}=1}^{l a-1} W_{i+k_{l}^{l a}, j+k_{W}^{l a}, c^{l a-1}}^{l a} * P_{n,+i, m+j, c} c^{l a-1}
$$

where $P_{n,+i, m+j, c^{l a-1}}$ is the input image, $B_{c} l a$ represents the bias of the current convolutional layer channel, $k_{l}^{l a}$ and $k_{w}^{l a}$ are respectively the height and width of the filter, la is the current layer, la- 1 is the previous layer, $W_{i+k_{l} c^{l a}, j+k_{w}, c^{l a-1}}$ is the kernel weights, and $P_{n, m, c}{ }^{l a}$ is the outcome of the convolutional layer at (n,m) pixel in $\mathrm{c}^{\text {la }}$ channel [12].

In this work, the parameters of this layer are selected according to the proposed pa-

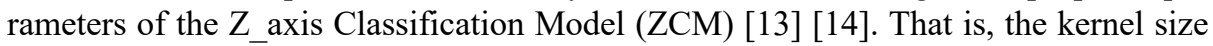
is used equal to $3 \times 3$ pixels, number of filters is equal to 2 filters, stride of 1 pixel and padding of $1 \mathrm{~s}$ surrounded pixels.

\subsection{ReLU layer}

ReLU is the second layer of the RDL and it has effective activation function that commonly used in the CNN. It does not pass the negative values of the previous layer. The following equation illustrates this activation function [15] [12]:

$$
O_{n, m, c} l a=\left\{\begin{array}{cc}
P_{n, m, c} l a-1 & \text { if } P_{n, m, c} l a-1 \\
0 & \text { otherwize }
\end{array}\right.
$$

where $O_{n, m, c} l a$ is an output of the ReLU layer. 


\subsection{Pooling layer}

It is a featured reduction layer. This layer reduces the feature map sizes that are provided by the ReLU layer. It can reduce the consumed computation time by taking the maximum values or average values of predefined pooling window. Its window strides across the ReLU feature maps until covering their employed information [16] [17] [12].

The parameters of the pooling layer here are also set according to the ZCM [13] [14]. That is, the maximum pooling type is selected, window size equal to $9 \times 9$ pixels, stride of 3 pixels and padding of 1 s surrounded pixels.

\subsection{Fully connected layer}

This layer has multiple tasks. It provides links between the previous feature maps and a single vector of nodes. It makes adaptation between the number of incoming nodes and required number of outgoing nodes, which can be equal to the number of output classes. It utilizes the following equation:

$$
F_{r}=\sum_{i=1}^{n_{1}^{l-1}} \sum_{j=1}^{n_{2}^{l-1}} \sum_{k=1}^{n_{3}^{l-1}} W_{i, j, k, r}^{l} * Q_{k, i, j} \quad 1 \leq r \leq n^{l}
$$

where $F_{r}$ is an outcome of the FC layer, $n_{1}^{l-1}$ and $n_{2}^{l-1}$ are respectively the height and width for a feature map of the pooling layer, $n_{3}^{l-1}$ represents the number of the generated feature maps in the pooling layer, $W_{i, j, k, r}^{l}$ are the weights of the FC layer and $Q_{k, i, j}$ represents a previous layer outcomes [12].

\subsection{Softmax layer}

It implements the softmax activation function which is represented by the following equation:

$$
S_{r}=\frac{\exp \left(F_{r}\right)}{\sum_{i=1}^{n^{l-1}} \exp \left(F_{i}\right)}
$$

where $S_{r}$ is an outcome of the softmax layer node [12].

\subsection{Classification layer}

A winner takes all rule is utilized in the classification layer using the following equation:

$$
D_{r}=\left\{\begin{array}{rr}
1 & \text { if } S_{r}=\max \\
0 & \text { otherwise }
\end{array}\right.
$$

where $D_{r}$ is an outcome of the classification layer and max is the maximum value in the Softmax layer [11]. 


\subsection{RDL feedback}

At the end of the training, the RDL stores two sets of weights. The first set of weights is for the FVs of index fingers and the second set of weights is for the FVs of middle fingers. For any testing, the RDL starts with the FV of an index finger with its set of weights. If the claimed verification does not match a certain class, the RDL switches to the FV of a middle finger with its set of weights. So, this feedback opportunity is taken over to further enhance the verification performance.

\section{Results and Discussions}

This section is composed of three parts. The first provides a brief description of the dataset utilized in this work. The second shows the performance of the proposed RDL method to verify subjects using their FVs. The last part demonstrates a comparison between different DL approaches using the same dataset that utilized in this work.

\subsection{Employed dataset}

The Hong Kong Polytechnic University Finger Image (PolyUFI) (Version 1.0) is may be the first dataset that acquires FV images. It contains of $6264 \mathrm{FV}$ images and finger texture (FT) images for 156 participants. The participants are from university campus and are from both gender who are aged under 30 years. Two sessions were organized to capture this dataset. The captured images are of type 24 bitmap images. Both index and middle finger were used. The dataset is for only fingers of left hands [18]

In this work, only FV images from the PolyUFI dataset have been exploited. Total of 2520 images have been employed for 105 participants, 1764 samples have been used for training and the remaining images (756) have been utilized for testing. The reason beyond selecting only 105 participants is that each one of these subjects has the full number of available FV images (12 FV images, 6 images acquired in session 1 and other 6 images captured in session 2).

\subsection{RDL performances}

The RDL has been trained for the FVs of index fingers and for the FVs of middle fingers. Two sets of weights have been saved, where the first set of weights are for the FV samples of index fingers and the second set of weights are for the FV samples of middle fingers. The utilized training parameters are as follows: optimizer of type Adaptive Moment Estimation (Adam), initial learning rate equal to 0.0001, gradient decay factor equal to 0.9 , squared gradient decay factor equal to 0.999 , epsilon equal to $10^{-8}$, mini-batch size epochs equal to 128 and max epochs equal to 100 .

The proposed RDL performances during the training phases show that the loss functions (or the error functions) are gradually decreased along iterations. This is true for training both the FVs of index fingers and FVs of middle fingers. Figure 2 shows the 
relationships between the loss functions and number of training iterations for the FVs of both index and middle fingers.

In the testing phase, a high accuracy of $91.19 \%$ has been benchmarked. The RDL can raise the testing performance from $76 \%$ for only the FVs of index fingers to $91.19 \%$ after applying the FVs of middle fingers. This enhancement can be explained by the ability of the RDL, which effectively employing supported data to improve the verification performances.

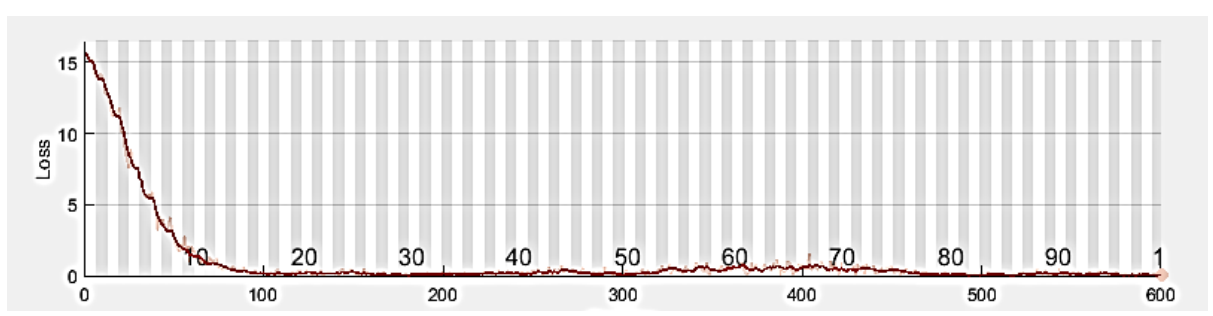

(a)

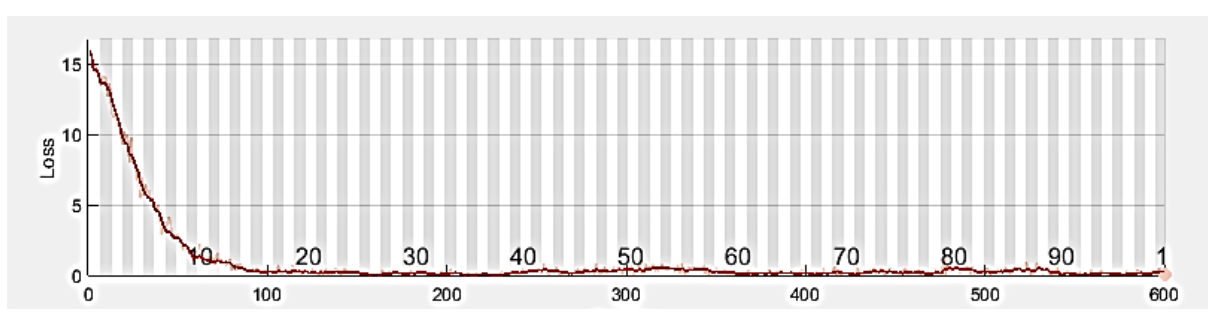

(b)

Fig. 2. RDL training curves, (a) training curve for the FV of index fingers and (b) training curve foOr the FV of middle fingers

\subsection{Comparisons}

The proposed RDL has further been examined by making comparisons with other methods. These methods include various state-of-the-art DL models. These models have been re-implemented using the dataset that is used in this work. The accuracies of these models compared to our suggested approach are given in Table 1.

In this table, it can be seen that the performances of other state-of-art DL models achieved low accuracies for both the FVs of index and middle fingers. This is due to their limitations in employing only a single information of the FV. Also, the results obviously show that the proposed RDL has reported the best performances. This can be explained by the ability of the RDL approach where it can effectively exploit both the FVs of index and middle fingers. 
Table 1. Comparisons between the accuracies of various state-of-the-art DL models

\begin{tabular}{|l|l|c|c|}
\hline \multicolumn{1}{|c|}{ Reference } & \multicolumn{1}{|c|}{ Deep Learning Model } & $\begin{array}{c}\text { Accuracies for the } \\
\text { FVs of index fingers }\end{array}$ & $\begin{array}{c}\text { Accuracies for the } \\
\text { FVs of middle fingers }\end{array}$ \\
\hline Omar et al. $[12]$ & $\begin{array}{l}\text { Deep Finger Texture Learning } \\
\text { (DFTL) }\end{array}$ & $62.38 \%$ & $61.43 \%$ \\
\hline Ibrahim et al. [19] & $\begin{array}{l}\text { Deep Fingerprint Classification } \\
\text { Network (DFCN) }\end{array}$ & $71.67 \%$ & $67.86 \%$ \\
\hline Albak et al. [20] & $\begin{array}{l}\text { Palm Convolutional Neural } \\
\text { Network (PCNN) }\end{array}$ & $71.19 \%$ & $72.38 \%$ \\
\hline AL-Hatab et al. $[13][14]$ & $\begin{array}{l}\text { Z axis Classification Model } \\
\text { (ZCM) }\end{array}$ & $76.90 \%$ & $75.24 \%$ \\
\hline Proposed Approach & RDL & \multicolumn{2}{|c|}{$91.19 \%$} \\
\hline
\end{tabular}

\section{Conclusion}

In this paper, a new version of the DL has been proposed. It is named the RDL. Furthermore, establishing a security method based on the FV can provide more secure recognition due to the FV characteristics. The proposed method was presented by the RDL that has FV backup information which can further enhance the performances of personal verification. The suggested RDL consists of multiple layers, global feedbacks and two sets of weights. It has been trained and tested for two FV samples of two fingers, the index and middle fingers. It can effectively utilize the FV characteristic of both fingers. It has benchmarked a high accuracy of $91.19 \%$. This is the biggest value compared to the accuracies of other various state-of-the-art DL models.

\section{$5 \quad$ References}

[1] E. Piciucco, E. Maiorana, C. Kauba, A. Uhl and P. Campisi, "Cancelable Biometrics for Finger Vein Recognition," IEEE, International Workshop on Sensing, Processing and Learning for Intelligent Machines (SPLINE), 2016. https://doi.org/10.1109/ splim.2016.7528396

[2] F. Tagkalakis, D. Vlachakis, V. Megalooikonomou and A. Skodras, "A Novel Approach To Finger Vein Authentication," IEEE International Symposium on Biomedical Imaging, 19 June 2017. https://doi.org/10.1109/isbi.2017.7950606

[3] T. Y. Hah, S.-L. Ju and K.-S. Kim, "Enhanced Approach for Finger Vein Recognition using BLPOC," IEEE SmartWorld, Ubiquitous Intelligence \& Computing, Advanced \& Trusted Computing, pp. 1188-1191, 2019. https://doi.org/10.1109/smartworld-uic-atc-scalcom-iopsci.2019.00221

[4] K. Shaheed, H. Liu, G. Yang, I. Qureshi, J. Gou and Y. Yin, "A Systematic Review of Finger Vein Recognition Techniques," Information, MDPI, 2018. https://doi.org/10.3390/ info9090213

[5] M. I. Obayya, M. El-Ghandour and F. Alrowais, "Contactless Palm Vein Authentication Using Deep Learning With Bayesian Optimization," IEEE access, vol. 9, January 5, 2021. https://doi.org/10.1109/access.2020.3045424 
[6] W. Liu, W. Li, L. Sun, L. Zhang and P. CHEN, "Finger Vein Recognition Based on Deep Learning," 12th IEEE Conference on Industrial Electronics and Applications (ICIEA), 2017. https://doi.org/10.1109/iciea.2017.8282842

[7] I. Boucherit, M. O. Zmirli, H. Hentabli and B. A. Rosdi, "Finger vein identification using deeply-fused Convolutional Neural Network," Journal of King Saud University - Computer and Information Sciences , 8 April 2020. https://doi.org/10.1016/j.jksuci.2020.04.002

[8] J. Zeng, F. Wang, J. Deng, C. Qin, Y. Zhai, J. Gan and V. Piuri, "Finger Vein Verification Algorithm Based on Fully Convolutional Neural Network and Conditional Random Field," IEEE access, vol. 8, April 17, 2020. https://doi.org/10.1109/access.2020.2984711

[9] S. Shendre and S. Sapkal, "A Hybrid Approach For Deep Learning Based Finger Vein Biometrics Template Security," International Journal Of Advance Scientific Research And Engineering Trends, vol. 5, no. 4, April 2020.

[10] W. Yang, S. Wang, J. Hu, G. Zheng, J. Yang and C. Valli, "Securing Deep Learning Based Edge Finger-vein Biometrics with Binary Decision Diagram," IEEE Transactions on Industrial Informatics, vol. 15, no. 7, July 2019. https://doi.org/10.1109/tii.2019.2900665

[11] R. Al-Nima, M. Al-Kaltakchi, S. Al-Sumaidaee, W. W. S. Dlay, T. Han and C. J., "Personal verification based on multi-spectral finger texture lighting images," IET Signal Processing, vol. 12, no. 9, 2018. https://doi.org/10.1049/iet-spr.2018.5091

[12] R. R. Omar, T. Han, S. A. M. Al-Sumaidaee and T. Chen, "Deep finger texture learning for verifying people," IET Biometrics, 2018. https://doi.org/10.1049/iet-bmt.2018.5066

[13] V. 8. p. 3.-3. 2. TEST Engineering \& Management, "Classifying Various Brain Activities By Exploiting Deep Learning Techniques And Genetic Algorithm Fusion Meth-od," TEST Engineering \& Management, vol. 83, pp. 3035-3052, 2020.

[14] M. M. AL-Hatab, R. R. O. Al-Nima, I. Marcantoni, C. Porcaro and L. Burattini, "Comparison Study Between Three Axis Views of Vision, Motor and Pre-Frontal Brain Activities," Journal of Critical Reviews, vol. 7, no. 5, pp. 2598-2607, 2020.

[15] A. Samreen, A. M. Taha, Y. V. Reddy and P. Sathish, "Brain Tumor Detection by Using Convolution Neural Network," International Journal of Online and Biomedical Engineering (iJOE), vol. 16, 2020. https://doi.org/10.3991/ijoe.v16i13.18545

[16] C. Cintas, M. Quinto-Sánchez and V. Acuña, "Automatic ear detection and feature ex-traction using Geometric Morphometrics and convolutional neural networks," IET Biometrics, vol. 6, pp. 211-223, 2017. https://doi.org/10.1049/iet-bmt.2016.0002

[17] L. Hou, Z. Li and H. Qu, "Fault Diagnosis of Rolling Bearing Using Wireless Sensor Networks and Convolutional Neural Network," International Journal of Online and Biomedical Engineering (iJOE, vol. 16, 2020. https://doi.org/10.3991/ijoe.v16i11.15959

[18] Hong Kong Polytechnic University, April 2009 - 2010. [Online]. Available: https://www4.comp.polyu.edu.hk/ csajaykr/fvdatabase.htm. [Accessed 20 jan 2021].

[19] A. M. Ibrahim, A. K. Eesee and R. R. O. Al-Nima, "Deep fingerprint classification network," TELKOMNIKA Telecommunication, Computing, Electronics and Control, vol. 19, 2021. https://doi.org/10.12928/telkomnika.v19i3.18771

[20] L. H. Albak, A. Hamed and R. R. O. Al-Nima, "Design Security System based on Arduino," Test Engineering and Management Journal, p. 82, 2020. 


\section{Authors}

Shaima Miqdad Mohamed Najeeb is presently one of the lecturers of technical engineering faculty, Computer Technical engineering department, Northern Technical University, Nenwa, Iraq. She received her B.E. degree in Computer Engineering in 1998 from Northern Technical University in Mosul, Iraq. Her M.Tech. degree in Computer Engineering focusing on "independent component analysis of EEG signal during various mental and movement tasks" IRAQ in 2013. Her current research interests include signal and image processing, EEG and biomedical signal processing, artificial intelligence. Al Mosul city - Hay AL Moharibin, Nenwa, Iraq. The number of articles in national databases is three. The number of articles in international databases is one.

Raid Rafi Omar Al-Nima received the BSc and MSc degrees in Technical Computer Engineering in 2000 and 2006, respectively. During 2006, he worked as an Assistant Lecturer in the Technical College of Mosul, Iraq. In 2011, he obtained the Lecturer scientific title in the same college. In 2017, he accomplished his PhD in the School of Electrical and Electronic Engineering at Newcastle University, UK. His research interests are in the fields of pattern recognition, security, artificial intelligence and image processing.

Mohand Lokman Aldabag completed his Ph.D. Program at Yasar University,Turkey. He received his B.E. in computer engineering degree from technical collegelMosul and his MSc. Degree in computer engineering from Mosul University, 1n 1998 and 2002 respectively. He worked at the department of technical computer engineering $\backslash$ Northern Technical university as an assistant lecturer in 2002-2012 and as a lecturer in the same university form 2012 till now. He published five articles in signal processing and hardware simulation of discrete wavelet. His research interests are biomedical signal processing, wavelet transform, and signal classification.

Article submitted 2021-06-08. Resubmitted 2021-06-28. Final acceptance 2021-06-29. Final version published as submitted by the authors. 